\title{
Ventilación no invasiva
}

\section{Non-invasive ventilation}

FABIO A. VARÓN V. ${ }^{(1)}$
En los últimos 20 años existe un interés creciente por el uso de la ventilación con presión positiva continua de la vía aérea y de aquella con presión positiva, con un aumento de las investigaciones y publicaciones sobre su utilización. Inicialmente, reportes y series de casos, estudios no controlados y finalmente estudios aleatorizados controlados, hacen mención a la selección de los pacientes, la selección de las interfaces o máscaras, los escenarios de uso clínico, los tipos de ventiladores y las ventajas de los diferentes equipos disponibles y protocolos de implementación son parte de la literatura disponible.

Una de las razones principales para considerar el uso de la ventilación no invasiva (VNI) es evitar las complicaciones asociadas a la intubación orotraqueal utilizando una máscara como alternativa de interfase entre el paciente y el ventilador mecánico, manteniendo la vía aérea intacta, preservando los mecanismos de defensa del tracto respiratorio y permitiéndole al paciente mantener la posibilidad de comunicarse de manera verbal, consumir alimentos o bebidas y manejar mejor las secreciones respiratorias.

La VNI inicialmente se usó en pacientes con exacerbaciones de enfermedad pulmonar obstructiva crónica (EPOC) con el objetivo de disminuir los niveles séricos de $\mathrm{CO}_{2}$, disminuir la carga sobre los músculos respiratorios, mejorar la ventilación alveolar y tratar de estabilizar el pH sanguíneo. Los buenos resultados en este grupo de pacientes, plantearon la ampliación de su uso a otras enfermedades en pacientes con falla respiratoria aguda, obteniendo resultados clínicos satisfactorios en algunos grupos específicos pero también demostrando que la principal causa de fracaso eran los grupos interdisciplinarios con preparación inadecuada.

A partir de este número se publicará una serie de revisiones acerca de las condiciones fisiológicas y fisiopatológicas que indican y explican el uso de este tipo de soporte pero que también demuestran que su uso inadecuado puede generar complicaciones tan graves como mortalidad.
(1)Fundación Neumológica Colombiana Correspondencia: Fabio A. Varón V., correo electrónico: fvaronvega@gmail.com Recibido: 10/03/16. Aceptado: 23/03/16. 